\title{
Improving the disease awareness: how a communication campaign brings hidradenitis suppurativa to the light
}

\author{
S. Ribero, ${ }^{1}$ P. Dapavo, ${ }^{1}$ C. Casalegno, ${ }^{2, \star}$ and the HS Awareness Working Group ${ }^{\dagger}$ \\ ${ }^{1}$ Department of Medical Sciences, Dermatologic Clinic, University of Turin, Turin, Italy \\ ${ }^{2}$ Department of Management, University of Turin, Turin, Italy \\ ${ }^{*}$ Correspondence: C. Casalegno. E-mail: cecilia.casalegno@unito.it
}

\begin{abstract} delayed therapy. association. and efficient the patient's management could be.

Received: 7 April 2019; Accepted: 17 May 2019

Conflict of interest

The disease awareness campaign was sponsored by AbbVie Italia.

Funding source

None.
\end{abstract}

Background Hidradenitis suppurativa is an inflammatory skin disease that presents a recurrence of painful and suppurating lesions in the apocrine gland-bearing regions, with a strong impact on the patients' life quality. Despite its peculiar presentation, early forms are often underestimated by patients and this would inevitably result in late diagnosis and

Objectives Improved communication around the disease could facilitate self-diagnosis and a quicker response from healthcare practitioners, especially in this moment when we dispose of effective treatment against this disease.

Methods A HS awareness campaign was conducted for 2 years with the help of a media agency and a patients'

Results Results confirm that a better communication has a strong impact on the disease awareness.

Conclusions This paper demonstrates that the more this disease awareness is carried on, the more quick, effective

\section{Introduction}

Hidradenitis suppurativa (HS) is an inflammatory skin disease with a characteristic clinical presentation of recurrent or chronic painful or suppurating lesions in the apocrine gland-bearing regions. ${ }^{1}$ The prevalence of $\mathrm{HS}$ is unknown and varies from $0.03 \%$ to $4 \% .^{2}$

Because of sparse epidemiologic data, disease burden of HS has not yet been established, and its actual prevalence worldwide is probably underestimated.

Surveys show that the mean delay in establishing a diagnosis of HS from the time of its initial presentation is 7.2 years. ${ }^{3}$ This may be due to insufficient awareness of HS and lower knowledge throughout the general population about this disease or to the fact that patients may accept recurrent symptoms that follow standard treatments and not seek further care.

The importance of effective communication has already been recognized by the medical literature during the last decades ${ }^{4-7}$;

${ }^{\dagger}$ HS Awareness Working Group members are listed in Appendix. the high value of the communication between the healthcare practitioner and the patient has been highlighted, ${ }^{8}$ together with the effectiveness of specific training courses to enhance healthcare practitioners' communication skills. Besides, a new value has been given to the so-called disease awareness, ${ }^{6-9}$ since the increase of screenings is of course fundamental, but it is also important to handle a communication which can lead to new diagnosis. ${ }^{6-8}$ This takes us to the aim of the present paper, which presents a new way to communicate with people through a comprehensive and integrated communication ${ }^{7-10}$ which takes advantage from the use of a more 'democratic' word, like 'cyst', also affecting the genital area, spread through social and mass media. ${ }^{10}$ This impacts on the HS awareness, which positively levers both on diagnosis skills and a more effective and efficient patient's management.

\section{Methods/the Italian HS awareness campaign}

The HS awareness campaign was conducted during a period of 2 years (2017 and 2018) by a media agency (Personal Media) with 


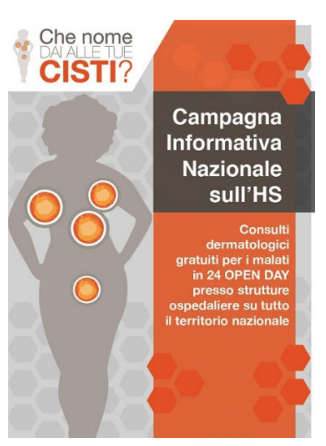

Figure 1 Flyer of the HS campaign 'Che nome dai alle tue cisti?' ('How do you name your cysts?').

the patronage of the patients' association Inversa Onlus. This campaign was intended to give the chance to exploit a free dermatologic screening reserved for people suffering from HS - or who think they may be suffering from this disease - and it took place on the whole Italian territory in hospitals and medical centres which joined this initiative. The communication around it was carried by using the more understandable term 'cyst', instead of the HS one (Fig. 1), and it was developed by integrating mass media (television, radio and local newspapers), social media (Facebook) and by continuously carrying out digital PR and institutional relations (with hospitals and medical diagnostic centres), whilst people could book, via telephone, a free screening during special and dedicated open days (34 in 2017 and 31 in 2018).

\section{Results and discussion}

As shown in Table 1, the target of the campaign was heterogenous, since during the open days the youngest person checked was 10 years old and the oldest was 78, and the M:F ratio was $38: 62$, confirming the existing literature. ${ }^{11}$ Regarding communication effectiveness, the 936 communication actions (546 in 2017 and 390 in 2018) were able to identify 555 new cases of HS (out of a potential 60000 derived from the 20 million Facebook users, considering a minimal prevalence of $0.03 \%$ ). Furthermore, the campaign was also useful to spread knowledge about HS: through the dedicated Facebook page and the information material (both printed and web-based) in which HS was described and explained, the selected users were able to consider whether they could be affected by HS. This approach resulted in a strong impact on visit requests by patients potentially suffering from HS and this is demonstrated by the 2017 and 2018 results: the first year 688 screening reservations led to 299 (43.45\% of the requests) new HS diagnoses, whilst the 463 screening reservations during the second year led to 256 (55.3\% of the requests) new diagnoses.

\section{Conclusion}

Results definitively confirm the literature ${ }^{6-9}$ and give an answer to the demand coming from previous studies ${ }^{8}$ : a better managed
Table 1 'Che nome dai alle tue cisti?' ('How do you name your cysts?') HS campaign: inputs and final results overview

\begin{tabular}{|c|c|c|c|}
\hline \multicolumn{4}{|l|}{ Organizing committee } \\
\hline Members & \multicolumn{3}{|c|}{ Activities' description } \\
\hline Inversa Onlus & \multicolumn{3}{|c|}{$\begin{array}{l}\text { As one of the major patients' association, } \\
\text { it supported the initiative and spread the } \\
\text { communication about it }\end{array}$} \\
\hline $\begin{array}{l}\text { Hospitals } \\
\text { Diagnostic centres }\end{array}$ & \multicolumn{3}{|c|}{$\begin{array}{l}\text { They both run the free screenings and did the } \\
\text { new diagnosis }\end{array}$} \\
\hline $\begin{array}{l}\text { Personal Media } \\
\text { agency }\end{array}$ & \multicolumn{3}{|c|}{$\begin{array}{l}\text { Run and organized the open days and all the } \\
\text { contacts with both the audience and the } \\
\text { doctors. It planned all the disease awareness } \\
\text { campaign and it made HS knowledge more } \\
\text { 'democratic', by using the common word 'cyst' }\end{array}$} \\
\hline \multicolumn{4}{|c|}{ ‘Che nome dai alle tue cisti?’ Campaign inputs } \\
\hline \multicolumn{2}{|l|}{2017} & \multicolumn{2}{|l|}{2018} \\
\hline $\begin{array}{l}\text { Communication } \\
\text { actions }\end{array}$ & Number & $\begin{array}{l}\text { Communication } \\
\text { actions }\end{array}$ & Number \\
\hline Campaign web page & 385 & Campaign web page & 268 \\
\hline TV and radio & 30 & TV and radio & 6 \\
\hline Online news & 33 & Online news & 28 \\
\hline Newspaper & 57 & Newspaper & 48 \\
\hline Agency web page & 21 & Agency web page & 19 \\
\hline Agency & 20 & Agency & 21 \\
\hline Total & 546 & Total & 390 \\
\hline \multicolumn{2}{|l|}{2017 - 34 stages report } & \multicolumn{2}{|l|}{2018 - 31 stages report } \\
\hline Total contacts & 2050 & Total contacts & 2383 \\
\hline Info requested via email & 91 & Info requested via email & 90 \\
\hline Phone calls & 1959 & Phone calls & 2293 \\
\hline Reservations & 688 & Reservations & 463 \\
\hline Screenings done & 629 & Screenings done & 417 \\
\hline HS new diagnosis & 299 & HS new diagnosis & 256 \\
\hline
\end{tabular}

communication has a strong impact on disease awareness and improved disease awareness allows the healthcare structures to better manage patients, with, for instance, the presence of specialized nurses and dedicated management teams. ${ }^{8}$ Furthermore, the integrated communication campaign, developed by using more understandable terminology, has improved the general awareness around HS, which is often misdiagnosed, and its daily care practices. As a result, the number of patients who take action could potentially increase substantially. One factor in whether or not patients seek help is the shame of the condition during intimacy. Privacy issues should be taken in account, as privacy policies on social media such as Facebook are very strict, and could potentially impact campaigns.

\section{References}

1 Saunte DML, Jemec GBE. Hidradenitis suppurativa: advances in diagnosis and treatment. JAMA 2017; 318: 2019-2032.

2 Calao M, Wilson JL, Spelman L et al. Hidradenitis suppurativa (HS) prevalence, demographics and management pathways in Australia: a population-based cross-sectional study. PLoS ONE 2018; 13(7): e0200683.

3 Saunte DM, Boer J, Stratigos A et al. Diagnostic delay in hidradenitis suppurativa is a global problem. Br J Dermatol 2015; 173: 1546-1549. 
4 Kurtz SM. Doctor-patient communication: principles and practices. Can J Neurol Sci 2002; 29(Suppl 2): S23-S29.

5 Thomas VJ, Cohn T. Communication skills and cultural awareness courses for healthcare professionals who care for patients with sickle cell disease. J Adv Nurs 2006; 53: 480-488.

6 Jacobsen GD, Jacobsen KH. Health awareness campaigns and diagnosis rates: evidence from National Breast Cancer Awareness Month. J Health Econ 2011; 30: 55-61.

7 Schiavo R. Health Communication: From Theory to Practice. John Wiley \& Sons, 2013.

8 Bettoli V, Pasquinucci S, Caracciolo S et al. The hidradenitis suppurativa patient journey in Italy: current status, unmet needs and opportunities. $J$ Eur Acad Dermatol Venereol 2016; 30: 1965-1970.

9 Long T, Taubenheim AM, Wayman J. The Heart Truth: using the power of branding and social marketing to increase awareness of heart disease in women. Soc Mar Q 2008; 14: 3-29.

10 Grilli R, Ramsay C, Minozzi S. Mass media interventions: effects on health services utilisation. Cochrane Database Syst Rev 2002; CD000389.

11 Garg A, Kirby JS, Lavian J, Strunk A. Sex- and age-adjusted population analysis of prevalence estimates for hidradenitis suppurativa in the United States. JAMA Dermatol 2017; 153: 760-764.

\section{Appendix}

HS Awareness Working Group: Mauro Alaibac (Dermatology Unit, University of Padova, Italy); Giuseppe Argenziano (Dermatology Unit, University of Campania, Naples, Italy); Nicola Giovanni Arpaia (Section of Dermatology, Miulli Regional Hospital, Bari, Italy); Giovanni Bagnoni (Dermatology Unit, Livorno Hospital, Livorno, Italy); Emilio Berti (Dermatologiy Unit, Fondazione IRCCS Ca' Granda Ospedale Maggiore Policlinico, Milan, Italy); Luca Bianchi (Department of Dermatology, University of Rome Tor Vergata, Rome, Italy); Daniela Bianchini (Department of Dermatology and Venereology, La Sapienza University of Rome, Rome, Italy); Piergiacomo Calzavara Pinton (Department of Dermatology, University of Brescia, Italy); Serafinella Patrizia Cannavò (Section of Dermatology, University of Messina, Italy); Maurizio Congedo (Section of Dermatology, Vito Fazzi Hospital, Lecce, Italy); Salvatore Corrao (Hospital Trust ARNAS Civico, Di Cristina, Benfratelli, Palermo); Antonio Costanzo (Dermatology Unit, Humanitas University and Humanitas Clinical and Research Center, Rozzano, Italy); Francesco Cusano (Department of Dermatology, G. Rummo Hospital, Benevento, Italy); Salvo Dattola (Tiberio Evoli Hospital, M. P. Salvo, Italy); Stefano Dattoli (Mater Domini Hospital, Catanzaro, Italy); Salvatore Deledda (Dermatology Clinic San Francesco Hospital ASL 3, Nuoro, Italy); Massimo Donini (Department of Dermatology, SS Giovanni e Paolo Civil Hospital, Venice, Italy); Gabriella Fabbrocini (Section of
Dermatology, Department of Clinical Medicine and Surgery, University of Naples Federico II, Naples, Italy); Maria Teresa Fierro (Dermatology Clinic, University of Turin, Italy); Caterina Foti (Dermatology Clinic, University of Bari, Italy); Antonia Gerarda Galluccio (Sacro Cuore Di Gesu Fbf Hospital, Benevento, Italy); Alessandro Gatti (Department of Dermatology, Hospital Ca' Foncello, Treviso, Italy); Carlo René Girardelli (Division of Dermatology, S. Chiara Hospital, Trento, Italy); Giampiero Girolomoni (Section of Dermatology and Venereology, University of Verona, Italy); Giuseppe Lemme (Carlo Urbani Hospital, Jesi, Italy); Piergiorgio Malagoli (Dermatology Unit, Azienda Ospedaliera San Donato Milanese, Milan, Italy); Giovanna Malara (Department of Dermatology, Grande Ospedale M. Morelli, Reggio Calabria, Italy); Santo Raffaele Mercuri (Dermatology Unit, IRCCS San Raffaele, Milan, Italy); Giuseppe Micali (Dermatology Clinic, University of Catania, Italy); Maria Antonia Montesu (Dermatology Unit, University of Sassari, Italy); Annamaria Offidani (Dermatology Unit, Polytechnic Marche University, Ancona, Italy); Manuela Papini (Dermatology Section, University of Perugia, Italy); Aurora Parodi (Di.S.Sal. Section of Dermatology, University of Genoa, San Martino Polyclinic Hospital, Genoa, Italy); Annalisa Patrizi (Division of Dermatology, University of Bologna, Italy); Giovanni Pellacani (Dermatology Unit, University of Modena and Reggio Emilia, Modena, Italy); Ketty Peris (Institute of Dermatology, Catholic University - Fondazione Policlinico Universitario A. Gemelli IRCCS, Rome, Italy); Angelo Piccirillo (San Carlo Hospital, Potenza, Italy); Paolo Pigatto (Dermatology Unit, IRCCS Istituto Ortopedico Galeazzi, University of Milan, Italy); Nicola Pimpinelli (Division of Dermatology, University of Florence, Italy); Concetta Potenza (Dermatology Unit 'Daniele Innocenzi', Sapienza University of Rome, Polo Pontino, Terracina, Italy); Eugenio Provenzano (Dermatology Unit, A.O. Cosenza, Italy); Marco Romanelli (Department of Dermatology, University of Pisa, Italy); Franco Rongioletti (Dermatology Clinic, University of Cagliari, Italy); Paola Savoia (Division of Dermatology, University of Eastern Piedmont, Novara, Italy); Marco Simonacci (Department of Dermatology, Macerata Hospital, Macerata, Italy); Riccardo Sirna (Dermatology Section, Misericordia Hospital, Grosseto, Italy); Giuseppe Stinco (Institute of Dermatology, 'Santa Maria della Misericordia' University Hospital, Udine, Italy); Giancarlo Valenti (Dermatology Unit, Pugliese Hospital, Catanzaro, Italy); Iris Zalaudek (Department of Dermatology, University of Trieste, Italy). 